\title{
Neural Coding of Tactile Decisions in the Human Prefrontal Cortex
}

\author{
Burkhard Pleger, ${ }^{1}$ Christian C. Ruff, ${ }^{1,3}$ Felix Blankenburg, ${ }^{1}$ Sven Bestmann, ${ }^{1,2}$ Katja Wiech, ${ }^{1,4}$ Klaas E. Stephan, ${ }^{1}$ \\ Almudena Capilla, ${ }^{1,5}$ Karl J. Friston, ${ }^{1}$ and Raymond J. Dolan ${ }^{1}$ \\ ${ }^{1}$ Wellcome Center of Neuroimaging and ${ }^{2}$ Sobell Department of Motor Neuroscience and Movement Disorders, Institute of Neurology, and ${ }^{3}$ Department of \\ Psychology, Institute of Cognitive Neuroscience, University College London, London WC1N 3AR, United Kingdom, ${ }^{4}$ Department of Human Anatomy and \\ Genetics, Oxford University, Oxford OX1 3QX, United Kingdom, and ${ }^{5}$ Center of Magnetoencephalography, Complutense University of Madrid, 28040 \\ Madrid, Spain
}

The neural processes underlying tactile decisions in the human brain remain elusive. We addressed this question in a functional magnetic resonance imaging study using a somatosensory discrimination task, requiring participants to compare the frequency of two successive tactile stimuli. Tactile stimuli per se engaged somatosensory, parietal, and frontal cortical regions. Using a statistical model that accounted for the relative difference in frequencies (i.e., Weber fraction) and discrimination accuracy (i.e., correct or incorrect), we show that trial-by-trial relative frequency difference is represented linearly by activity changes in the left dorsolateral prefrontal cortex (DLPFC), the dorsal anterior cingulate cortex, and bilateral anterior insular cortices. However, a circumscribed region within the left DLPFC showed a different response pattern expressed as activity changes that were monotonically related to relative stimulation difference only for correct but not for incorrect trials. Our findings suggest that activity in the left DLPFC encodes stimulus representations that underlie veridical tactile decisions in humans.

Key words: tactile decision; tactile discrimination; Weber fraction; somatosensory cortex; dorsolateral prefrontal cortex; functional magnetic resonance imaging

\section{Introduction}

Decisions based on perceptual properties of stimuli rely on several processing stages involving sensation, perception, and action. In everyday life, these processes are intertwined, and we can switch between them with remarkable speed and flexibility (Miller and Cohen, 2001). Although the sensory stages of perceptual decisions have attracted attention since the early days of cognitive neuroscience (Newsome et al., 1989; Schall and Bichot, 1998), a more recent focus has been on processes that bridge sensation and action. An increasing focus on decision making is now of interest to psychologists, economists, and neuroscientists (Glimcher and Rustichini, 2004).

Candidate regions mediating a transformation of sensation to action have been identified within a frontoparietal network, where neurons are thought to encode abstract information related to perceptual decisions (Heekeren et al., 2004; Machens et al., 2005). In monkeys, decision-related signals have been studied

Received Aug. 17, 2006; revised 0ct. 28, 2006; accepted 0ct. 30, 2006.

This work was supported by a grant from the Wellcome Trust Programme (R.J.D.). C.C.R., F.B., S.B., K.E.S., and K.J.F. were supported by the Wellcome Trust, K.W. was supported by German Research Society Grant Wi 1957/2-1, and A.C. was supported by the Community of Madrid. We thank Raffael Kalisch, Dharshan Kumaran, Hakwan Lau, and Jeremie Mattout for fruitful discussions and Peter Aston and Eric Featherstone for technical support. We also thank two unknown reviewers for their helpful contributions.

Correspondence should be addressed to Burkhard Pleger, Wellcome Department of Imaging Neuroscience, Institute of Neurology, University College London, 12 Queen Square, London WC1N 3BG, UK. E-mail: b.pleger@fil.ion.ucl.ac.uk.

DOI:10.1523/JNEUROSCI.4275-06.2006

Copyright $\odot 2006$ Society for Neuroscience $\quad$ 0270-6474/06/2612596-06\$15.00/0 in great detail in the tactile domain (Romo et al., 1993, 1999, 2002, 2004; Hernandez et al., 2000, 2002; de Lafuente and Romo, 2002; Romo and Salinas, 2003). As in other sensory systems, the cortical representations of the sensory stimulus may inform perceptual decisions (Platt and Glimcher, 1999; Shadlen and Newsome, 2001; Machens et al., 2005).

Ernst Heinrich Weber developed an approach to the study of human perception for physical stimuli in a quantitative manner. One of his key observations, often referred to as Weber's law, was that perception of a stimulus change depends on both the magnitude of the change $(\Delta s)$ and the stimulus baseline intensity $(s)$. At threshold, the ratio of stimulus change and baseline intensity $(\Delta s / s)$ is constant (Weber, 1834). Successful discrimination of two stimuli will thus depend on the relative stimulus change, $\Delta s / s$ (Dayan and Abbott, 2001). In this view, correct perceptual decisions depend on how well two stimuli can be reliably discriminated. The underlying neuronal mechanisms for this decision process are mostly unknown.

We used event-related functional magnetic resonance imaging (fMRI) while subjects performed a frequency discrimination task adapted from previous monkey experiments (Romo et al., 1999). In our task, the difficulty of discriminating the two frequencies (coded as Weber fractions) is the crucial variable that describes the subjects' ability to make correct decisions. We manipulated this difficulty variable to study cortical representations for correct and incorrect decisions. Within a single trial, the putative processes involve subjects perceiving a first stimulus [fre- 
quency 1 (f1)], holding it in working memory, and executing a decision by comparing it with a subsequent second stimulus (f2) (Machens et al., 2005).

To assess decision-related activation, we focused on stimulusevoked responses to the second tactile stimulus of each pair as a function of the corresponding Weber fraction. We asked whether the relative stimulus difference $(\Delta s / s)$ is encoded by responses within distinct brain regions as a function of decision accuracy (i.e., correct or incorrect discrimination). We then assessed whether this evaluation engages distinct regions within the parietal cortex known to be involved in tactile discrimination [primary (SI) and secondary somatosensory cortex (SII)] (Golaszewski et al., 2006; Pleger et al., 2006) or in frontal regions that may represent task-independent contributors to behavioral control (Ridderinkhof et al., 2004).

\section{Materials and Methods \\ Subjects}

The study was approved by the Joint Ethics Committee of the Institute of Neurology and National Hospital for Neurology and Neurosurgery London and was performed in accordance with the Declaration of Helsinki (1964). Ten right-handed healthy subjects (six males; age, 20-37 years; mean age, $29 \pm 6.5$ years) gave their written, informed consent and underwent two test sessions before they performed the same task during two fMRI sessions.

\section{Event-related $f M R I$}

Functional and structural brain scans were acquired on a 3T head scanner (Magnetom Allegra; Siemens, Erlangen, Germany). We acquired blood oxygenation level-dependent (BOLD) volumes with a gradientecho $\mathrm{T} 2^{*}$-weighted echo-planar imaging sequence (echo time, $30 \mathrm{~ms}$; repetition time, $2.21 \mathrm{~s}$; flip angle, $90^{\circ}$ ). The thickness of each volume was $2 \mathrm{~mm}$ with a $3 \times 3 \mathrm{~mm}^{2}$ in-plane resolution and a slice distance of $1 \mathrm{~mm}$. Each volume consisted of 34 oblique slices (transversal-coronal tilt, $-10^{\circ}$ ), which covered the entire brain, excluding the cerebellum. A total of 519 volumes per session were acquired continuously. We also acquired a high-resolution anatomical image using an isotropic threedimensional spoiled gradient-recalled acquisition in a steady-state sequence with 107 sagittal-orientated slices covering the entire brain. We used structural images across subjects to form a mean group image. For the spatial assignment of functional signal changes, results obtained from group analysis were superimposed onto this structural mean image.

\section{Frequency discrimination task}

The task was the same as in a previous study (Pleger et al., 2006). In brief, subjects performed a two-alternative forced-choice frequency discrimination task. The task consisted of 70 events and 14 so-called "null events." These null events had the same duration as "real" events but did not comprise tactile stimulation. The sequence of the events was chosen randomly. During the task, subjects were instructed to fixate a small cross in the center of a screen. Meanwhile, they discriminated between the frequency of two electrical stimuli ( $\mathrm{f} 1$ and $\mathrm{f} 2$ ) applied sequentially to the right index finger (see Fig. 1, top). We used a Digitimer (Hertfordshire, UK) DS7A stimulator for electrical finger stimulation. Disposable surface-adhesive electrodes (Spes Medica, Battipaglia, Italy) were mounted on the radial side of the right index finger, with the cathode to the distal phalanx and the anode to the proximal phalanx. Stimulation intensity was adjusted to 2.5 times the sensory threshold. Each stimulus lasted for $1 \mathrm{~s}$ and was separated by an interstimulus interval of 2-4 s (randomly jittered in steps of $1 \mathrm{~s}$ ). The full range of both frequencies was between 20 and $36 \mathrm{~Hz}$. The absolute difference between the two frequencies for each event was 1-7 Hz. For each session, a total of 10 events were presented for each frequency difference, resulting in 70 events. Three to $5 \mathrm{~s}$ after application of the second stimulus (in $1 \mathrm{~s}$ steps), subjects had to indicate within $2 \mathrm{~s}$ whether the first or the second frequency was higher by pressing a button with the left (nonstimulated) index finger. The motor response was triggered by an " $r$ " that replaced the fixation cross until the subjects pressed the button. They were instructed to press the button once for the first stimulus or twice for the second stimulus. No feedback was given. Two to $3 \mathrm{~s}$ after the response interval (randomly jittered in $1 \mathrm{~s}$ steps), the first stimulus of the next event was delivered.

Perceptual accuracy was expressed in percentage of correct discriminations across all similarity levels $(|\mathrm{f} 1-\mathrm{f} 2|=1-7 \mathrm{~Hz})$. To assess whether the subjects' performance (i.e., percentage of correct responses) was correlated with our difficulty variable (i.e., the Weber fraction of the frequency difference), we used the Pearson correlation analysis. The Weber fraction $(\Delta s / s)$ was computed as the ratio of change in frequency (i.e., high - low frequency) relative to the intensity of the lower frequency (Nieder and Miller, 2003).

\section{Data analysis}

Functional neuroimaging. The data were preprocessed and analyzed using the statistical parametric mapping (SPM) software package (SPM2; Wellcome Department of Imaging Neuroscience, University College London, London, UK). We discarded the first six volumes during which BOLD signal reached steady state. The remaining 513 volumes entered preprocessing. Movement artifacts were removed using realignment and unwarping (Andersson et al., 2001). Volumes were spatially normalized to the standard template of the Montreal Neurological Institute (MNI; voxel size, $2 \mathrm{~mm}^{3}$ ) (Friston et al., 1995). Finally, we smoothed the volumes using a $10 \mathrm{~mm}$ (full-width half-maximum) isotropic, threedimensional Gaussian filter.

To assess the effect of frequency discrimination per se, we distinguished between trials and null events at the first (within-subject) level in several stick functions encoding the presentation of stimuli. The first stimulus of each pair was represented by one stimulus function. For the second stimulus, we used two stimulus functions that coded the onsets of stimuli that were correctly or incorrectly discriminated. In addition, two separate regressors (i.e., one each for correct and incorrect discriminations) represented the parametric modulation of the stimulus functions for the second stimulus with the difficulty variable of present interest (i.e., with the relative perception of the frequency difference between both stimuli coded as a Weber fraction, $\Delta s / s)$. This variable was computed as the ratio of the change in frequency (i.e., high - low frequency) relative to the intensity of the lower frequency (Nieder and Miller, 2003). The motor response after the second stimulus was represented by another stimulus function. All stimulus functions were convolved with a hemodynamic response function to form regressors for our design matrix (i.e., convolution model).

By specifying contrasts on the associated parameter estimates, we were able to test for the main effect of discrimination accuracy (i.e., correct or incorrect), the main effect of relative stimulus difference (i.e., Weber fraction, averaged over correct and incorrect discriminations), and finally the interaction between accuracy and relative stimulus difference (i.e., the difference in activity specific to the relative stimulus difference, for correct vs incorrect trials).

All of our inferences were made at the second (between-subject) level by entering the appropriate contrast into one-way ANOVA. The effect of frequency discrimination was assessed by comparing the real events and null events across subjects using the one-way ANOVA (threshold, $p=$ 0.0001 , uncorrected). The main effect of our difficulty variable (i.e., Weber fraction) is shown in Figure 3 at two thresholds, $p=0.0001$ uncorrected and $p=0.05$ family-wise error corrected across the entire brain. The interaction between Weber fraction and accuracy is shown in Figure 4 at $p=0.001$ uncorrected and $p=0.05$ family-wise error corrected across the entire brain. All reported coordinates correspond to the anatomical MNI space as used in SPM2.

To assess the orthogonality between the regressors, in other words the independence between conditions (first stimulus, second stimulus correct discrimination, second stimulus incorrect discrimination, motor response), we averaged the correlation coefficients between conditions across the two sessions. These values were then averaged across subjects. In fact, by modeling first and second stimuli separately for correct and incorrect discriminations, our regression became decorrelated [first vs second stimulus correct, $r=0.16 \pm 0.03$ (average $\pm \mathrm{SD}$ ); first vs second stimulus incorrect, $r=0.09 \pm 0.04$; second stimulus correct vs motor 

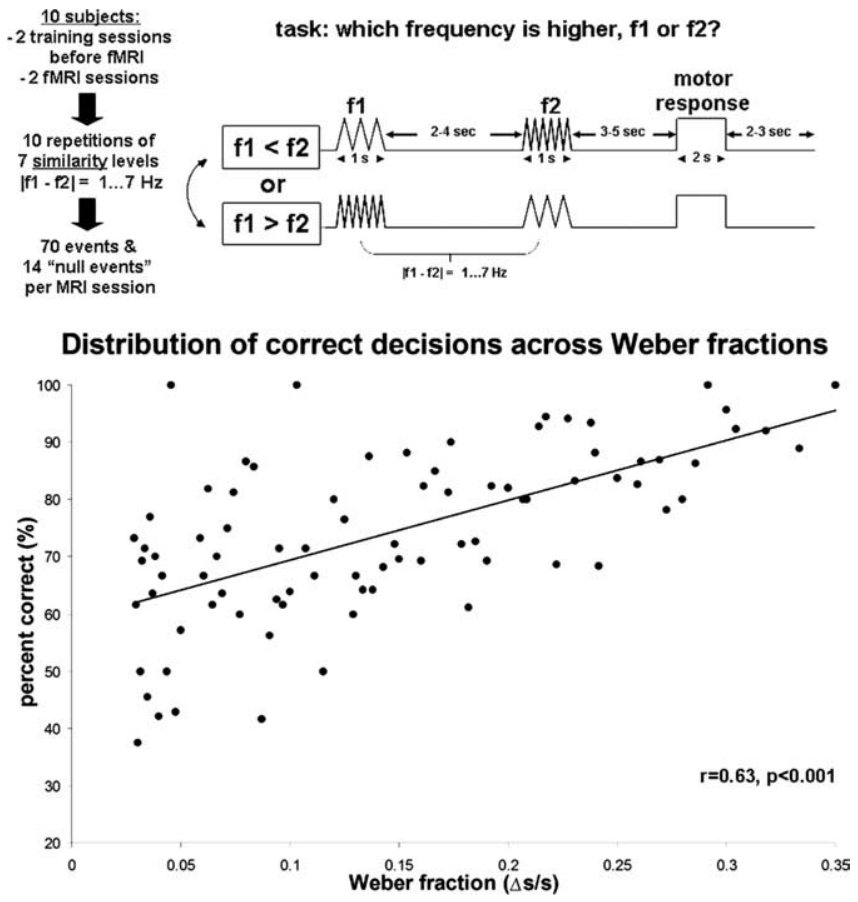

Figure 1. Frequency discrimination task and perceptual performance. Top, Schematic drawing of one single trial of the task. Within each trial, subjects received two frequency stimulations ( $f 1$ and $\mathrm{f} 2$ ) to the right index finger, each for $1 \mathrm{~s}$, separated by a variable interstimulus interval. The range of frequencies for both stimuli was between 20 and $36 \mathrm{~Hz}$. The application of $\mathrm{f} 2$ was followed by a delay period. Then subjects had to indicate within $2 \mathrm{~s}$ whether $\mathrm{f} 1$ or $\mathrm{f} 2$ was higher by pressing a button with the left (nonstimulated) index finger once for $\mathrm{f} 1$ or twice for $\mathrm{f} 2$. Bottom, Distribution of correct decisions across the Weber fractions $(\Delta s / s ; \Delta s=$ high - low frequency $=1 \ldots 7 \mathrm{~Hz} ; s=$ lower frequency $=20 \ldots 36 \mathrm{~Hz}$ ). Correlation analysis showed that subjects made more correct decisions for large (e.g., $\Delta s=7 \mathrm{~Hz}, s=20 \mathrm{~Hz}$ ) relative to small frequency differences in proportion to the lower frequency (e.g., $\Delta s=1 \mathrm{~Hz}, s=20 \mathrm{~Hz}$ ).

response, $r=0.24 \pm 0.02$; second stimulus incorrect vs motor response, $r=0.13 \pm 0.05]$.

\section{Results}

\section{Frequency discrimination task}

Subjects first practiced the frequency discrimination task in two consecutive test sessions to achieve a stable baseline performance before the two fMRI sessions (mean correct responses across all stimuli \pm SE: first test session, $73.62 \pm 3.2 \%$; second test session, $72.94 \pm 2.68 \%$; fMRI, $75.88 \pm 2.16 \%$ across both sessions). The range of both frequencies within each session was between 20 and $36 \mathrm{~Hz}$. We included seven levels of absolute stimulus difference between the first and the second stimulus of each trial $(\Delta s=$ lowest. . . highest frequency $=1 . .7 \mathrm{~Hz}$ ) but coded them as relative stimulus differences " $\Delta s$ " in proportion to different background intensities " $s$ " (i.e., as Weber fractions; see Materials and Methods). We randomly varied the frequency for both stimuli for each subject, which guaranteed a stable attention level throughout each session. However, for each subject, the number of presentations of each frequency was identical during two consecutive test sessions and the after two fMRI sessions.

First, we tested whether the subjects' performance (i.e., percentage of correct discriminations) was correlated with our difficulty variable (i.e., Weber fraction). Pearson correlation analysis confirmed that our difficulty variable was indeed a meaningful characterization of behavioral performance (i.e., percentage of correct discriminations) (Fig. 1, bottom). Subjects made more correct decisions for large (e.g., $\Delta s=7 \mathrm{~Hz}, s=20 \mathrm{~Hz}$ ) relative to small (e.g., $\Delta s=1 \mathrm{~Hz}, s=20 \mathrm{~Hz}$ ) frequency differences, in proportion to the base frequency $\left(r=0.63 ; p=2.6 \times 10^{-10}\right)$.

\section{Event-related fMRI}

Cortical responses to tactile discrimination per se were estimated using a standard event-related linear convolution model comparing discrimination trials with null trials (see Materials and Methods). In line with recent findings, we found activations in SI (Recanzone et al., 1992; Hernandez et al., 2000; de Lafuente and Romo, 2002; Harris et al., 2002) and SII (Francis et al., 2000; Romo et al., 2002) contralateral to the stimulated index finger, as well as supplementary motor area/dorsal cingulate cortex (Romo et al., 1993), premotor cortex (Hernandez et al., 2002; Romo et al., 2004), posterior parietal cortex (Grefkes and Fink, 2005), insular cortex (Pleger et al., 2006), and prefrontal cortex (Romo et al., 1999) in both hemispheres (Fig. 2) (supplemental Table 1, available at www.jneurosci.org as supplemental material).

To assess differences in cortical activation between correct and incorrect discriminations (main effect of discrimination accuracy), we compared correct and incorrect responses to the second stimulus regardless of our difficulty variable (i.e., Weber fraction). No brain area showed such a significant effect of accuracy per se in this comparison. We next examined the main effect of our difficulty variable (i.e., the impact of Weber fraction on BOLD responses), regardless of whether responses were correct or incorrect. Crucially, for each subject, trials with correct and incorrect responses covered the full range of similarity levels $(\Delta s)$. We found positive correlations in the left dorsolateral prefrontal cortex (DLPFC), in the dorsal anterior cingulate cortex (dACC), and in the anterior insula cortices (Fig. 3).

Finally, we tested whether the relationship between BOLD responses and Weber fraction show different expressions for correct compared with incorrect discriminations, as indicated by a significant interaction of accuracy and relative frequency difference. A single region in the left DLPFC showed such a discriminatory pattern, with a linear increase in activity with relative frequency difference for correct (mean contrast estimate $\pm 90 \%$ confidence interval, $14.22 \pm 2.94 ; t=7.96$ ) but not for incorrect decisions $(-1.73 \pm 2.94 ; t=0.97)$ (Fig. 4). We found no brain regions that expressed the opposite pattern, a larger correlation between BOLD responses and relative stimulus difference for incorrect trials compared with correct trials.

\section{Discussion}

The goal of this study was to investigate the neural processes underlying perceptual decisions in the tactile domain, using a two-alternative forced-choice frequency discrimination task. Specifically, we asked which brain areas encode the following: (1) the discrimination accuracy, (2) the relative frequency difference (i.e., Weber fraction), and critically, (3) the interaction between accuracy and relative frequency difference. In our task, the crucial difficulty variable is the relative frequency difference, coded as a Weber fraction [i.e., as the difference between two successive stimuli $(\Delta s)$ in proportion to the magnitude of the base frequency $(s)$ ] (Weber, 1834). We found that this difficulty variable of relative frequency was positively correlated with the percentage of correct discriminations and with activity in the left DLPFC (Brodmann's area 10). Interestingly, this parametric response profile of the left DLPFC was expressed specifically for correct but not for incorrect judgments.

In perceptual two-alternative decisions, it is assumed that the decision is the result of continuously accumulating noisy stimulus information until one of the two response criteria is reached 


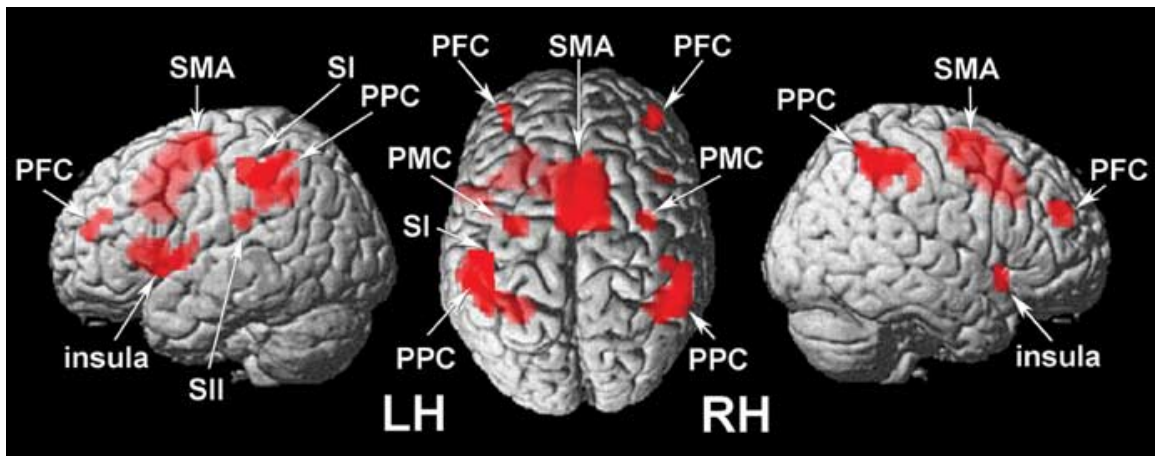

Figure 2. Cortical regions involved in frequency discrimination (contrast: discrimination trials $>$ "null trials"). Significant signal changes were found in the SI and SII contralateral to the stimulated index finger. In addition, we found activation in the supplementary motor cortex (SMA), the premotor cortex (PMC), the posterior parietal cortex (PPC), the anterior insula, and the prefrontal cortex (PFC) of both hemispheres (MNI coordinates and $t$ scores are listed in supplemental Table 1, available at www.jneurosci.org as supplemental material). LH, Left hemisphere; $\mathrm{RH}$, right hemisphere.

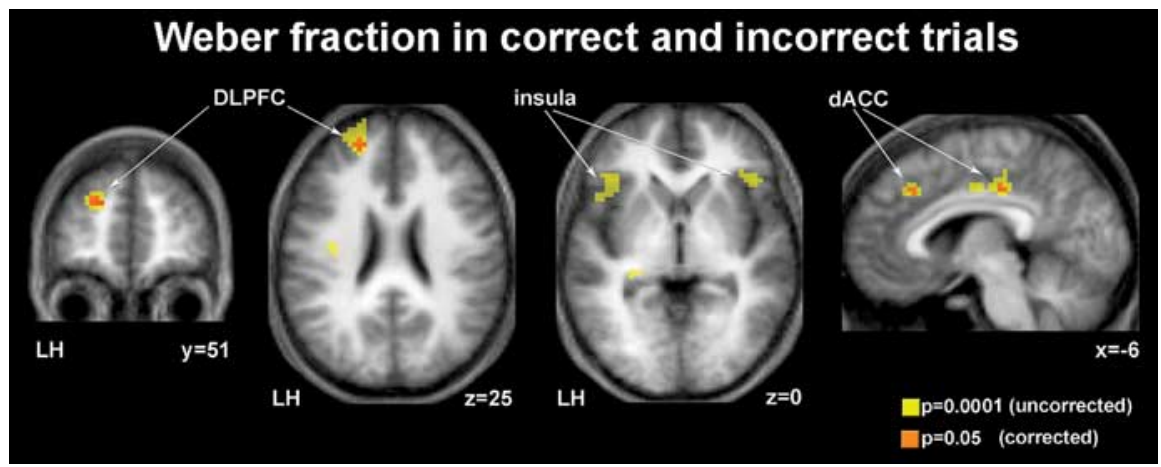

Figure 3. Cortical regions showing activity increases as a function of the relative frequency difference (i.e., main effect of Weber fraction, contrast: Weber fraction across correct and incorrect discriminations $>$ baseline). All activations are projected on a coronal (left), axial (middle), and sagittal (right) MRI slice. LH, Left hemisphere. Weighing the stimulus function of all trials according to the relative frequency difference (i.e., Weber fraction) revealed activation in the left DLPFC $[-21,51,24(x, y, z) ; t=$ 6.4; Brodmann's area 10], the dACC $[-9,30,39(x, y, z)$; Brodmann's area 6; $t=7.24 ;$ and $-6,-24,39(x, y, z) ;$ Brodmann's area $32 ; t=5.72]$, and in both anterior insular cortices [LH: $-51,15,-18(x, y, z) ; t=6.08$; right hemisphere: $48,27,-3(x, y, z)$; $t=5.17]$. In all plots, yellow regions show activity thresholded at $p=0.0001$ uncorrected, whereas red regions show the corresponding activity at $p=0.05$, family-wise error corrected across the entire brain.

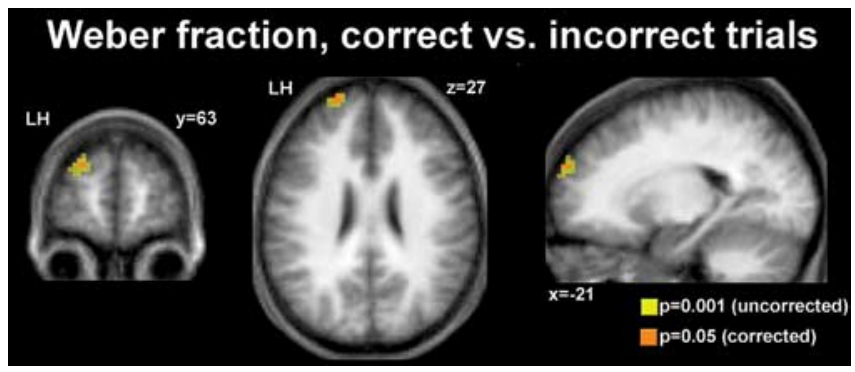

Figure 4. Cortical regions coding the relative frequency difference (i.e., Weber fraction) for correct compared with incorrect discriminations (interaction contrast: Weber fraction $\times$ correct trials $>$ Weber fraction $\times$ incorrect trials). All activations are projected onto a coronal (left), axial (middle), and sagittal (right) MRI slice. LH, Left hemisphere. The figure shows a region in the DLPFC [Brodmann's area 10; peak voxel: $-21,60,27(x, y, z) ; t=6.31 ; p=0.005$ family-wise error corrected across the entire brain] that showed positive correlations with the Weber fraction of the stimulus difference only for correct (mean contrast estimate $\pm 90 \%$ confidence interval, $14.22 \pm 2.94 ; t=7.96)$ but not for incorrect discriminations $(-1.73 \pm$ $2.94 ; t=0.97)$.

(Smith and Ratcliff, 2004). Our finding that the level of activity in the DLPFC encoded the relative stimulus difference (i.e., our difficulty variable) suggests that this region may accumulate critical decision-relevant stimulus information. It is of interest that decision-relevant stimulus representations in tactile discriminations engage the left DLPFC, a region that is often assumed to contribute to behavioral control (Kerns et al., 2004; Ridderinkhof et al., 2004; Rushworth et al., 2004).

In monkeys, neuronal recordings from cells within a frontoparietal network during discrimination of frequency differences have provided insights into the configuration of the evoked neuronal responses during tactile decisions. The firing rate of neurons within this network during stimulus periods contains sufficient information necessary to solve the task (Romo et al., 1993, 1999, 2004; Hernandez et al., 2000, 2002). Although the presence of tactile stimuli per se activated a similar frontoparietal network described by these monkey experiments, we found signal changes correlated with the difficulty variable Weber fraction, regardless of whether trials were correct or not, in the left DLPFC, in the bilateral insular cortices, and in the dACC. The latter activation was expressed in Brodmann's area 6/32, a region thought to index preresponse conflicts (Ridderinkhof et al., 2004). This suggests that these activations may relate to the encoding of increasing uncertainty about the decision.

Several complementary accounts ascribe specific functions to the ACC (and its anatomical subdivisions) (Ridderinkhof et al., 2004) as mediating error detection (Falkenstein et al., 1991; Dehaene et al., 1994; Holroyd et al., 1998), detection of behavioral conflicts (Botvinick et al., 1999; Mayr et al., 2003), or control of effort in self-generated acts (Kerns et al., 2004). The DLPFC, on the other hand, is believed to encode on-line information necessary for perceptual identification (Everling et al., 2002; Heekeren et al., 2004) and generation of a subsequent appropriate response (Paus et al., 1993; Paus, 2001). Task-related interactions between the functions of these medial and lateral frontal structures have been reported in lesion patients (Gehring and Knight, 2000) and healthy human subjects (Kerns et al. 2004), indicating that interactions between the DLPFC and the ACC may both contribute to behavioral control and guidance (Ridderinkhof et al., 2004).

In addition to the dACC, we found activations related to the relative frequency difference (for both correct and incorrect discriminations) within the insula in both hemispheres. An anatomical feature of the somatosensory system and insular cortex is the complex network of fibers that link functional subregions (Burton and Kopf, 1984), although specific knowledge about these pathways in humans is limited. In monkeys, a ventrally directed tactile processing pathway leads from SI to the frontal cortex and the temporal lobe limbic structures via relays in SII and the insular cortex (Friedman et al., 1986; Preuss and Goldman-Rakic, 1989).

In touch or pain perception, the insula has been described as part of an "interoceptive" network that may code distinct aspects 
of the physiological condition of all body tissues. This supports awareness of feeling states from the body (like the sense of touch), which may be important for higher-level body representations (Craig, 2002; Critchley et al., 2005). In the present study, we found that the anterior insula increased its activity linearly with the relative frequency difference. This suggests that sensory input, first processed in somatosensory cortices (Craig, 2003), may be encoded and remapped within the insula (Burton and Sinclair, 2000) to contribute to a neuronal representation of crucial stimulus information.

Our key observations are that trial-by-trial relative stimulus differences (i.e., Weber fractions) are represented linearly by activity in the left DLPFC, dACC, and bilateral anterior insular cortices, whereas a spatially distinct area within the DLPFC showed an interaction of accuracy and relative stimulation difference. Activity in this DLPFC region increased linearly with the relative frequency differences only for correct but not for incorrect discriminations (Fig. 4). In our task, for any single trial, subjects first perceive a stimulus and hold it in working memory (Machens et al., 2005). It has been shown in previous monkey studies that during this period, the firing rate of neurons in the inferior convexity of the prefrontal cortex monotonically relates to the maintained stimulus frequency. This suggests a basic representation of one-dimensional sensory stimulus quantities in working memory (Romo et al., 1999). In our task, subjects were required to make a decision based on comparing the second stimulus against the memory trace left by the first stimulus (Brody et al., 2002). During this process, we found that BOLD responses in the left DLPFC linearly increased as a function of the relative frequency difference but only for correct decisions. This supports the idea that correct perceptual decisions depend on the accumulation of meaningful stimulus information until sufficient information for a response is obtained (Ratcliff and Hacker, 1981; Gold and Shadlen, 2001; Smith and Ratcliff, 2004; Philiastides et al., 2006). In the incorrect trials, we found no relationship between BOLD responses and the relative frequency difference. This suggests that errors originate from the inability to generate an adequate representation of stimulus frequency. We conclude that the findings highlight a crucial role for the left DLPFC in encoding the relative frequency difference of tactile stimuli that inform correct tactile decisions. More generally, the findings indicate a central contribution of the DLPFC to sensory-based decision making.

\section{References}

Andersson JL, Hutton C, Ashburner J, Turner R, Friston K (2001) Modeling geometric deformations in EPI time series. NeuroImage 13:903-919.

Botvinick M, Nystrom LE, Fissell K, Carter CS, Cohen JD (1999) Conflict monitoring versus selection-for-action in anterior cingulate cortex. $\mathrm{Na}-$ ture 402:179-181.

Brody CD, Hernandez A, Zainos A, Lemus L, Romo R (2002) Analysing neuronal correlates of the comparison of two sequentially presented sensory stimuli. Philos Trans R Soc Lond B Biol Sci 357:1843-1850.

Burton H, Kopf EM (1984) Ipsilateral cortical connections from the second and fourth somatic sensory areas in the cat. J Comp Neurol 225:527-553.

Burton H, Sinclair RJ (2000) Attending to and remembering tactile stimuli: a review of brain imaging data and single-neuron responses. J Clin Neurophysiol 17:575-591.

Craig AD (2002) How do you feel? Interoception: the sense of the physiological condition of the body. Nat Rev Neurosci 3:655-666.

Craig $\mathrm{AD}$ (2003) Interoception: the sense of the physiological condition of the body. Curr Opin Neurobiol 13:500-505.

Critchley HD, Rotshtein P, Nagai Y, O’Doherty J, Mathias CJ, Dolan RJ (2005) Activity in the human brain predicting differential heart rate responses to emotional facial expressions. NeuroImage 24:751-762.

Dayan P, Abbott LF (2001) Neural encoding I: firing rates and spike statis- tics. In: Theoretical neuroscience (Dayan P, Abbott LF, eds), pp 18-19. Cambridge, MA: MIT.

Dehaene S, Posner MI, Tucker DM (1994) Localization of a neural system for error detection and compensation. Psychol Sci 5:303-305.

de Lafuente V, Romo R (2002) A hidden sensory function for motor cortex. Neuron 36:785-786.

Everling S, Tinsley CJ, Gaffan D, Duncan J (2002) Filtering of neural signals by focused attention in the monkey prefrontal cortex. Nat Neurosci 5:671-676.

Falkenstein M, Hohnsbein J, Hoormann J, Blanke L (1991) Effects of crossmodal divided attention on late ERP components. II. Error processing in choice reaction tasks. Electroencephalogr Clin Neurophysiol 78:447-455.

Francis ST, Kelly EF, Bowtell R, Dunseath WJ, Folger SE, McGlone F (2000) fMRI of the responses to vibratory stimulation of digit tips. NeuroImage 11:188-202.

Friedman DP, Murray EA, O’Neill JB, Mishkin M (1986) Cortical connections of the somatosensory fields of the lateral sulcus of macaques: evidence for a corticolimbic pathway for touch. J Comp Neurol 252:323-347.

Friston KJ, Ashburner J, Frith CD (1995) A spatial registration and normalization of images. Hum Brain Mapp 3:165-189.

Gehring WJ, Knight RT (2000) Prefrontal-cingulate interactions in action monitoring. Nat Neurosci 3:516-520.

Glimcher PW, Rustichini A (2004) Neuroeconomics: the consilience of brain and decision. Science 306:447-452.

Golaszewski SM, Siedentopf CM, Koppelstaetter F, Fend M, Ischebeck A, Gonzalez-Felipe V, Haala I, Struhal W, Mottaghy FM, Gallasch E, Felber SR, Gerstenbrand F (2006) Human brain structures related to plantar vibrotactile stimulation: a functional magnetic resonance imaging study. NeuroImage 29:923-929.

Gold JI, Shadlen MN (2001) Neural computations that underlie decisions about sensory stimuli. Trends Cogn Sci 5:10-16.

Grefkes C, Fink GR (2005) The functional organization of the intraparietal sulcus in humans and monkeys. J Anat 207:3-17.

Harris JA, Miniussi C, Harris IM, Diamond ME (2002) Transient storage of a tactile memory trace in primary somatosensory cortex. J Neurosci 22:8720-8725.

Heekeren HR, Marrett S, Bandettini PA, Ungerleider LG (2004) A general mechanism for perceptual decision-making in the human brain. Nature 431:859-862.

Hernandez A, Zainos A, Romo R (2000) Neuronal correlates of sensory discrimination in the somatosensory cortex. Proc Natl Acad Sci USA 97:6191-6196.

Hernandez A, Zainos A, Romo R (2002) Temporal evolution of a decisionmaking process in medial premotor cortex. Neuron 33:959-972.

Holroyd CB, Dien J, Coles MG (1998) Error-related scalp potentials elicited by hand and foot movements: evidence for an output-independent errorprocessing system in humans. Neurosci Lett 242:65-68.

Kerns JG, Cohen JD, MacDonald III AW, Cho RY, Stenger VA, Carter CS (2004) Anterior cingulate conflict monitoring and adjustments in control. Science 303:1023-1026.

Machens CK, Romo R, Brody CD (2005) Flexible control of mutual inhibition: a neural model of two-interval discrimination. Science 307:1121-1124.

Mayr U, Awh E, Laurey P (2003) Conflict adaptation effects in the absence of executive control. Nat Neurosci 6:450-452.

Miller EK, Cohen JD (2001) An integrative theory of prefrontal cortex function. Annu Rev Neurosci 24:167-202.

Newsome WT, Britten KH, Movshon JA (1989) Neuronal correlates of a perceptual decision. Nature 341:52-54.

Nieder A, Miller EK (2003) Coding of cognitive magnitude: compressed scaling of numerical information in the primate prefrontal cortex. Neuron 37:149-157.

Paus T (2001) Primate anterior cingulate cortex: where motor control, drive and cognition interface. Nat Rev Neurosci 2:417-424.

Paus T, Petrides M, Evans AC, Meyer E (1993) Role of the human anterior cingulate cortex in the control of oculomotor, manual, and speech responses: a positron emission tomography study. J Neurophysiol 70:453-469.

Philiastides MG, Ratcliff R, Sajda P (2006) Neural representation of task difficulty and decision making during perceptual categorization: a timing diagram. J Neurosci 26:8965-8975. 
Platt ML, Glimcher PW (1999) Neural correlates of decision variables in parietal cortex. Nature 400:233-238.

Pleger B, Blankenburg F, Bestmann S, Ruff CC, Wiech K, Stephan KE, Friston KJ, Dolan RJ (2006) Repetitive transcranial magnetic stimulationinduced changes in sensorimotor coupling parallel improvements of somatosensation in humans. J Neurosci 26:1945-1952.

Preuss TM, Goldman-Rakic PS (1989) Connections of the ventral granular frontal cortex of macaques with perisylvian premotor and somatosensory areas: anatomical evidence for somatic representation in primate frontal association cortex. J Comp Neurol 282:293-316.

Ratcliff R, Hacker MJ (1981) Speed and accuracy of same and different responses in perceptual matching. Percept Psychophys 30:303-307.

Recanzone GH, Merzenich MM, Jenkins WM, Grajski KA, Dinse HR (1992) Topographic reorganization of the hand representation in cortical area $3 \mathrm{~b}$ owl monkeys trained in a frequency-discrimination task. J Neurophysiol 67:1031-1056.

Ridderinkhof KR, Ullsperger M, Crone EA, Nieuwenhuis S (2004) The role of the medial frontal cortex in cognitive control. Science 306:443-447.

Romo R, Salinas E (2003) Flutter discrimination: neural codes, perception, memory and decision making. Nat Rev Neurosci 4:203-218.

Romo R, Ruiz S, Crespo P, Zainos A, Merchant H (1993) Representation of tactile signals in primate supplementary motor area. J Neurophysiol 70:2690-2694.

Romo R, Brody CD, Hernandez A, Lemus L (1999) Neuronal correlates of parametric working memory in the prefrontal cortex. Nature 399:470-473.

Romo R, Hernandez A, Zainos A, Lemus L, Brody CD (2002) Neuronal correlates of decision-making in secondary somatosensory cortex. Nat Neurosci 5:1217-1225.

Romo R, Hernandez A, Zainos A (2004) Neuronal correlates of a perceptual decision in ventral premotor cortex. Neuron 41:165-173.

Rushworth MF, Walton ME, Kennerley SW, Bannerman DM (2004) Action sets and decisions in the medial frontal cortex. Trends Cogn Sci 8:410-417.

Schall JD, Bichot NP (1998) Neural correlates of visual and motor decision processes. Curr Opin Neurobiol 8:211-217.

Shadlen MN, Newsome WT (2001) Neural basis of a perceptual decision in the parietal cortex (area LIP) of the rhesus monkey. J Neurophysiol 86:1916-1936.

Smith PL, Ratcliff R (2004) Psychology and neurobiology of simple decisions. Trends Neurosci 27:161-168.

Weber EH (1834) De pulso, resorptione, auditu et tactu. In: Annotationes anatomicae et physiologicae. Leipzig, Germany: Koehler. 